\title{
Clinical experience of functional electrical stimulation in complete paraplegia
}

\author{
Yoichi Shimada, Kozo Sato, Eiji Abe, Hitoshi Kagaya, Kunio Ebata, Masashi Oba and Mineyoshi Sato \\ Department of Orthopaedic Surgery, Akita University School of Medicine, 1-1-1 Hondo, Akita 010, Japan
}

\begin{abstract}
Percutaneous intramuscular electrodes and a portable multichannel system were used to restore the function of the paralyzed lower extremities in six patients with complete paraplegia. The total number of inserted electrodes was 168. All of the patients could stand, two could walk in parallel bars, and two could walk with a walker. The rate of breakage of electrodes was only $0.6 \%$ in our series. There were $10(6.0 \%)$ superficial infections, and 10 $(6.0 \%)$ movement of electrodes which required reimplantation. The results suggest that the ultrafine intramuscular electrode is practical for long term use with paraplegic patients. Although the system can be used for paraplegic patients during the activities of daily living, it will be necessary to develop a closed-loop controller to reduce the amount of stimulation to the extensor muscles and extend the endurance of upright activity to reduce fatigue.
\end{abstract}

Keywords: functional electrical stimulation (FES); paraplegia; standing; walking

\section{Introduction}

Recent advances in computer technology have made it possible to control paralyzed muscles by electrical stimulation. Functional electrical stimulation (FES) has been investigated in some laboratories as a method of achieving standing and walking, and some investigators have already reported some success in restoring ambulatory function for people who are paraplegic. ${ }^{111,12}$ The Sendai group and the Nippon Electric Company have developed the FES system with percutaneous electrodes, ${ }^{3-7}$ and this has been available in our Department and has been used to restore locomotion in complete paraplegic patients since 1990. ${ }^{14}$ Here we describe the clinical results and problems arising with FES.

\section{Materials and methods}

The subjects included six males with complete deficit of spinal cord function, with no motor function or sensation below the level of injury. The average patient age was 36.5 years (range, 19-68 years). The cause of the spinal cord lesion in two patients was a spinal arteriovenous malformation at T12 level. The other four subjects had sustained a spinal cord injury at the levels of T12, T6, T5 and T8. The average time since injury was 1.8 years (range, 3 months to 5 years), and the average follow-up period was 4.4 years (range, 2 years 11 months to 5 years 4 months (Table 1).

The FES system developed by the Sendai Group

Correspondence: Y Shimada and the Nippon Electric Company was used in this study. This system consists of a stimulation data creating system (SDC) and a 30-channel stimulator. The SDC was used to compose and store the stimulation parameters which set the threshold voltages for each muscle, and controlled the pulse shape and the individual pulse sequence (Figure 1). The portable stimulator measured $8.9 \times 14.5 \times 3.1 \mathrm{~cm}$ and weighed $330 \mathrm{~g}$, and was usually worn on a belt (Figure 2).

The indwelling electrode was formed as a helically wound Teflon-coated 19 strand stainless steel wire (Nippon Seisen Co. Ltd.) (Figure 3). The tip of the electrode was deinsulated. Electrodes were implanted percutaneously into the motor point of the muscles.

The pulse amplitude was modulated from 0 to -15 V. Rectangular pulse trains were used with 200 microseconds pulse width and a pulse interval of $50 \mathrm{~ms}$. A customized pattern of stimulation was composed for each subject based on the observed muscle function.

Prior to implantation, the sensitivity and location of motor points was determined using surface electrode stimulation. Implantation of the electrodes was performed under sterile conditions. We chose a common body-entry point for electrodes located at both anterior proximal thighs (Figure 4). The number of percutaneous electrodes was 29 in case 1, 19 in case 2, 22 in case 3,30 in case 4,34 in case 5 and 34 in case 6 , totalling 168 . The stimulated muscles or nerves are as described in Table 2. Mediolateral support of the ankle was provided with an ankle-foot orthosis, if considered to be necessary. 
Table 1

\begin{tabular}{|c|c|c|c|c|c|c|c|c|c|}
\hline \multirow[b]{2}{*}{ Case } & \multirow[b]{2}{*}{$\begin{array}{l}\text { Age } \\
(y / o)\end{array}$} & \multirow[b]{2}{*}{ Sex } & \multirow[b]{2}{*}{ Disease } & \multirow[b]{2}{*}{$\begin{array}{l}\text { Level of } \\
\text { injury. }\end{array}$} & \multirow[b]{2}{*}{$\begin{array}{c}\text { Duration } \\
\text { from onset }\end{array}$} & \multirow[b]{2}{*}{$\begin{array}{c}\text { Follow-up } \\
\text { time }\end{array}$} & \multirow[b]{2}{*}{$\begin{array}{l}\text { Number of } \\
\text { electrodes }\end{array}$} & \multicolumn{2}{|c|}{ Acquired abilities } \\
\hline & & & & & & & & $\begin{array}{l}\text { Standing } \\
(\min )\end{array}$ & $\begin{array}{c}\text { Walking } \\
(\mathrm{m})\end{array}$ \\
\hline 1 & 68 & male & AVM* & $\mathrm{T} 12$ & 5 years & 5 years & 29 & 2 & 0 \\
\hline 2 & 22 & male & $\mathrm{SCI} * *$ & $\mathrm{~T} 12$ & 4 months & 5 years & 19 & 15 & 10 \\
\hline 3 & 61 & male & AVM & $\mathrm{T} 12$ & 2 years & 3 years 3 months & 22 & 5 & 0 \\
\hline 4 & 19 & male & SCI & T6 & 3 months & 4 years 7 months & 30 & 20 & 20 \\
\hline 5 & 25 & male & SCI & $\mathrm{T} 5$ & 1 year 10 months & 5 years 5 months & 34 & 15 & 10 \\
\hline 6 & 24 & male & SCI & T8 & 1 year 4 months & 2 years 11 months & 34 & 20 & 20 \\
\hline
\end{tabular}

*Arteriovenous malformation. ${ }^{* *}$ Spinal cord injury

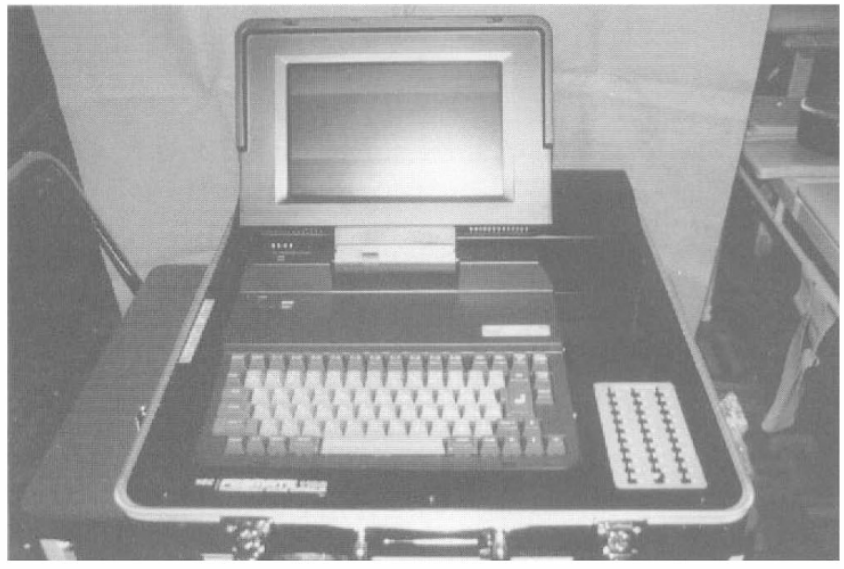

Figure 1 Stimulation data creating system (SDC). The SDC is used to compose and store the stimulation parameters that permit the threshold voltages for muscle, and control pulse shape and pulse sequence

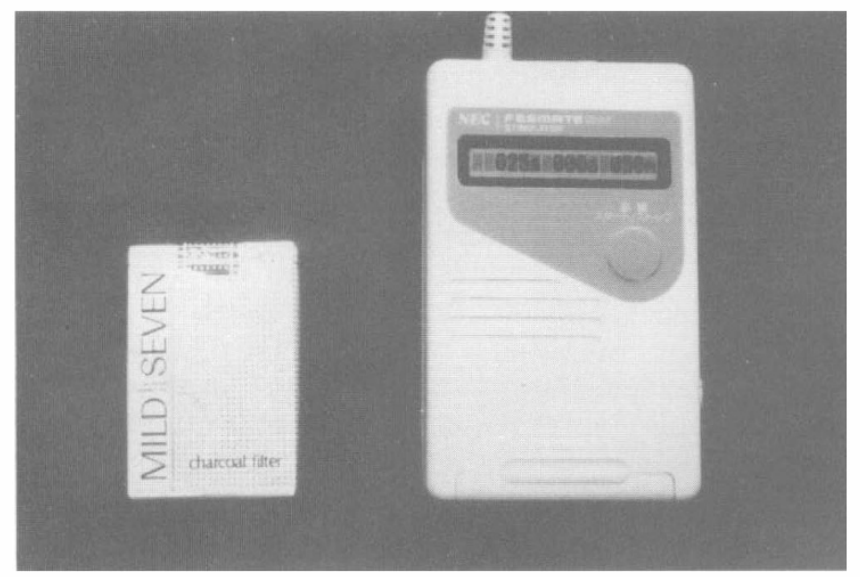

Figure 2 30-channel portable stimulator. The portable stimulator measures $8.9 \times 14.5 \times 3.1 \mathrm{~cm}$ and weighs $330 \mathrm{~g}$, and is usually worn on a belt

Electrical exercise commenced 2 weeks after implantation to allow time for the tissues to heal. This reduced the problem associated with electrode movement. Stimulation of the muscles was conducted for 10 min three times daily at the beginning and was increased every two weeks by an extra $10 \mathrm{~min}$. After

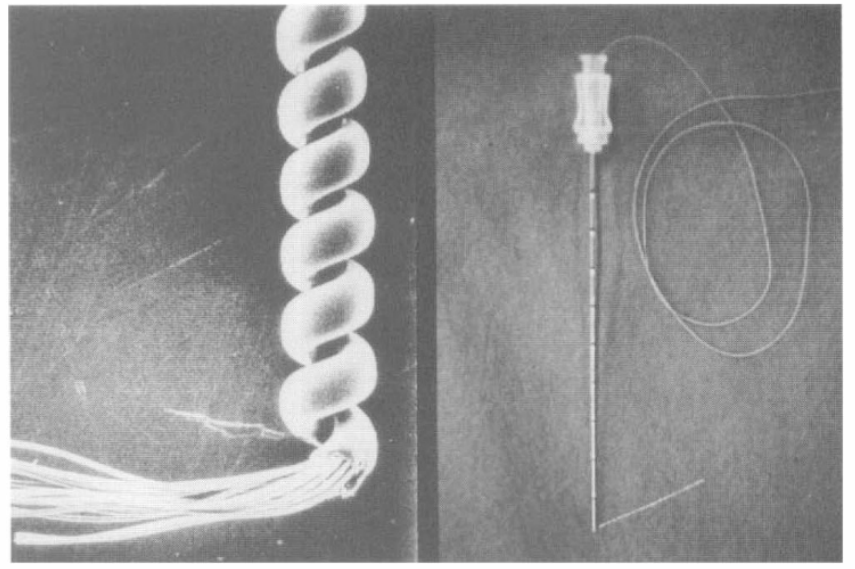

Figure 3 Percutaneous electrode. The indwelling electrode is formed as a helically wound Teflon-coated 19 strand stainless steel wire. The tip of the electrode is deinsulated

10 weeks, the stimulation was applied for $60 \mathrm{~min}$, three times daily. After exercising like this for more than three months, standing and walking training in the parallel bars started.

\section{Results}

The volume of the muscles measured by $\mathrm{CT}$ scan increased with daily electrical stimulation in many muscles. ${ }^{9}$ In others which failed to increase their volume we suspected peripheral nerve damage. The muscle conditioning stimulation was continued to reduce muscle fatigue. The use of the withdrawal reflex was beneficial in assisting flexion of the hip joint. The skin complexion of the legs in all cases turned well. They became less susceptible to cold. In two patients their decubitus was improved by the increased blood circulation.

All six patients could stand, two walked in parallel bars with AFO's and two walked with a walker. The maximum duration of standing by electrical stimulation was $2 \mathrm{~min}$ in case $1,15 \mathrm{~min}$ in case $2,5 \mathrm{~min}$ in case 3,20 $\mathrm{min}$ in case 4,15 $\mathrm{min}$ in case 5 and $20 \mathrm{~min}$ in case 6 . The maximum distance of walking was 10 meters in case 2 and case 5 in parallel bars, 20 meters 
in case 4 (Figure 5) and case 6 with walker (Table 1). One of the reasons for discontinuing standing and walking was fatigue of the knee extensors. A short double-limb support was required during walking in patients 2, 4 and 6 .

Only one electrode broke $(0.6 \%)$, in patient 4 , at the site deep in the iliopsoas muscle. Loss of sufficient contraction force due to movement of electrodes required 10 electrodes to be reimplanted $(6.0 \%)$. On ten occasions $(6.0 \%)$ there was a superficial infection around the site of electrode insertion. These infections were treated by topical sterilization of the skin and oral antibiotics. Removal of electrodes was not necessary, and there were no visceral complications in our series.

\section{Discussion}

Potentially there are many benefits for paraplegic patients from FES standing. Standing may help to prevent joint contractures by eliminating the chronic sitting posture, and it may reduce osteoporosis via weight bearing. The upright posture may improve the position of the internal organs, and aid bowel and bladder function. It may also reduce the chance of pressure sores by relief of pressure, and could aid circulation. Increased functional abilities while standing may enhance personal self-esteem. ${ }^{16}$

The bilateral knee-ankle-foot orthoses (KAFO) has traditionally been used for standing in complete paraplegic patients, but there is high rejection rate because of the difficulties in donning and doffing orthotic devices and the significant energy expenditure of walking. ${ }^{16}$

In 1960 , Kantrowitz ${ }^{10}$ reported that a paraplegic patient could stand by use of electrical stimulation. Restoration of function of the paralyzed extremities has been remarkably developed in recent years. Marsolais ${ }^{11,12}$ reported a large number of paraplegic patients who achieved standing and walking.

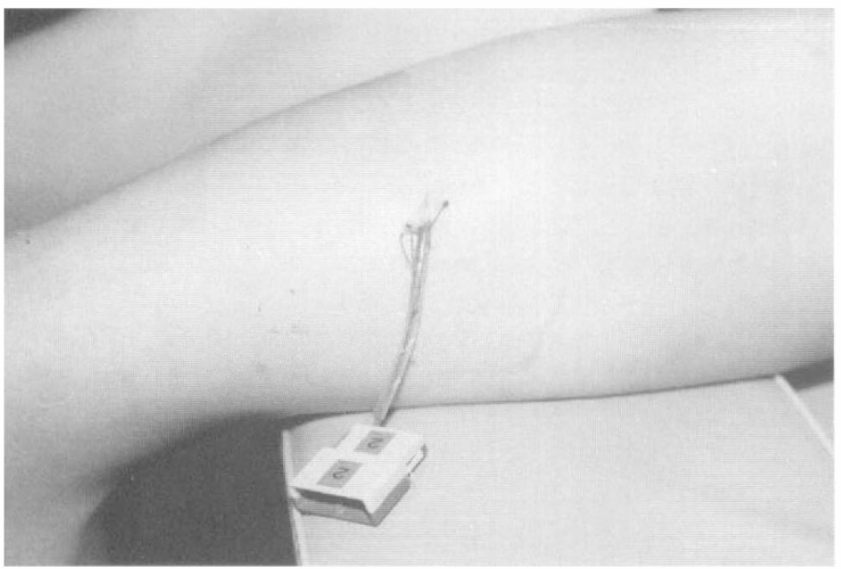

Figure 4 Body-entry point for electrodes. A common bodyentry point for electrodes located in both anterior proximal thighs
Overall, applications for FES are for eating, writing, make-up, standing, walking, posture control, turning over in bed, urination and penile erection. Indications for FES are as follows: (1) upper motor neuron disorders; (2) absence of contractures and of ossification of the joints; (3) without severe osteoporosis; (4)

Table 2 Stimulated muscles and nerves

\begin{tabular}{ll}
\hline Joint function & Muscles and nerves \\
\hline $\begin{array}{c}\text { Hip flexion } \\
\text { extension }\end{array}$ & Iliopsoas m. \\
abduction & Gluteus maximus m. \\
Knee flexion & Hamstrings \\
extension & Quadriceps m., Femoral n. \\
Ankle dorsiflexion & Common peroneal n. \\
plantarflexion & Gastrocnemius m., Posterior tibial n. \\
eversion & Peroneus longus m. \\
Trunk extension & Spinal erector m. \\
\hline m:
\end{tabular}

m: muscle; n: nerve

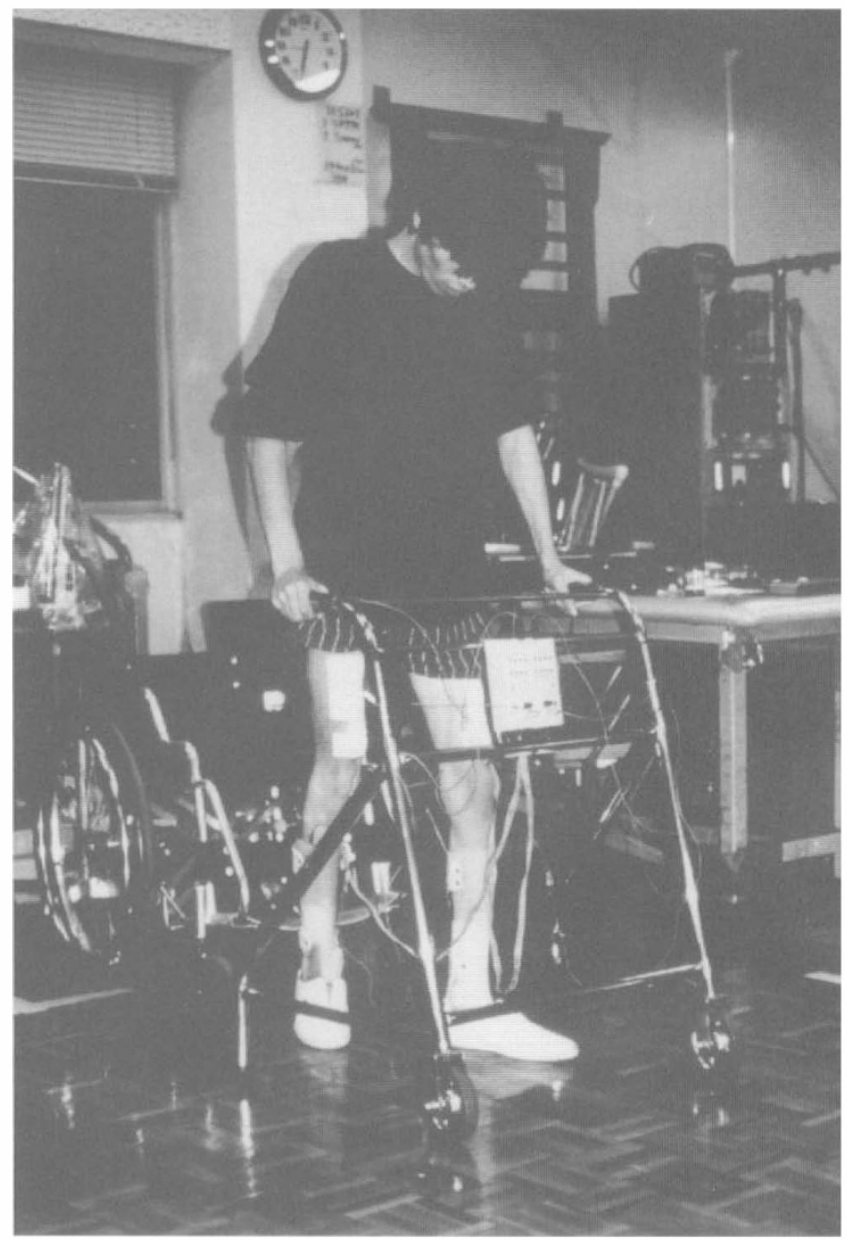

Figure 5 Standing and walking in case 4 (T6 complete paraplegia). Maximum duration of standing by electrical stimulation is $20 \mathrm{~min}$. Walking with walker and original closed-loop control system. Maximum distance of walking is 20 meters 


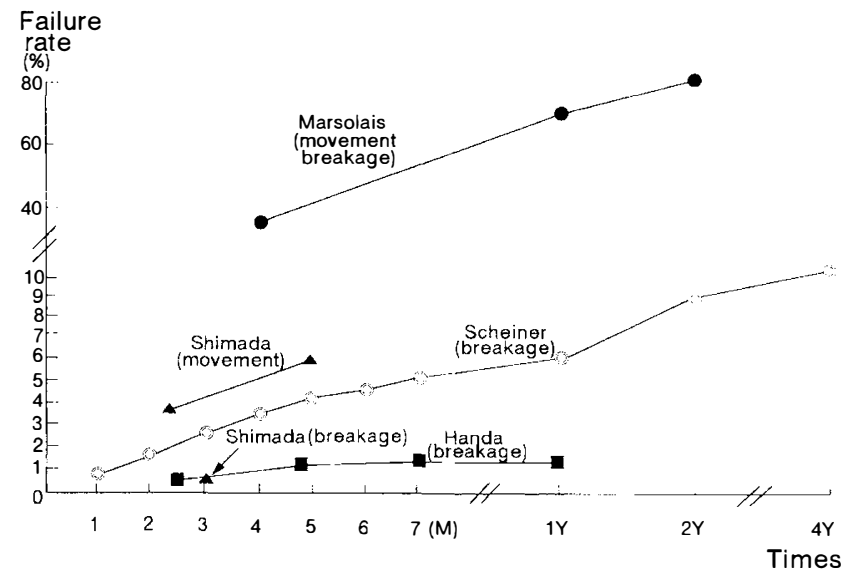

Figure 6 The rate of breakage and movement of percutaneous electrodes. The ultrafine intramuscular electrode (Nippon Seisen Co. Ltd.) is practical and is available for long time use

absence of skin problems; (5) normal balance; (6) a normal emotional state. On the other hand, contraindications for FES are as follows: (1) severe deformities and contractures of the joints; (2) extensive peripheral nerve injuries; (3) severe muscle atrophy; (4) severe ectopic ossifications; (5) severe visceral disorders; (6) obesity; (7) inadequent balance.

There are three stimulation methods-surface electrodes, percutaneous electrodes and implanted electrodes. Surface electrodes require daily placement and removal. In addition, some patients feel discomfort when excessive stimulation is given by surface electrodes. It is difficult to stimulate the deep muscles, such as the iliopsoas muscle, by surface electrodes. Implanted electrodes need reoperation when electrodes break or move. Previously used percutaneous electrodes failed at a high rate. Marsolais $^{11}$ reported that $35 \%$ failed within 4 months, and only $30 \%$ continued to function 1 year later, with $20 \%$ surviving for 2 years, and giving an average failure rate of 2.5 electrodes per month in a 48 electrode system that required replacement. Since the result of the clinical use of this coil electrode was frustrating because of the frequent necessity to replace implanted electrodes which became dysfunctional, Scheiner et al. developed a double helix electrode. ${ }^{13}$ A total of 22 paralyzed human subjects received implants of 775 double helix electrodes. In which 453 electrodes $(65 \%)$ continued to produce strong and stable muscle contractions. The oldest implanted electrode has remained functional for 4.5 years. The main causes for failure of double helix electrodes have been: (1) inability to locate and properly place the electrode at a suitable site for stimulation $(28,4 \%)$; (2) unwanted changes in muscle response to stimulation $(91,12 \%)$; (3) increase in electrode impedance $(74,10 \%$; assumed breakage); (4) intolerable pain during stimulation $(8,1 \%) ; \quad(5)$ infection $(4,0.5 \%)$. This double helix electrode design has proved to be practical to obtain chronic stimulation of selected muscles as compared with the coil wire electrode. However, the failure rate of the double helix electrode remains high $(35 \%)$ for use in daily living. In our series the rate of breakage and the movement of electrodes was only $6.5 \%$ for an average of 2.2 years. Handa $^{3}$ reported that the rate of breakage was $1.3 \%$ in 457 electrodes using the same electrodes as ours (Figure 6). A helically coiled structure of the electrode has demonstrated resistance to breakage even when the electrode was implanted into bulky leg muscles. ${ }^{4-6}$ These results suggested that the ultrafine intramuscular electrode is practical and available for long term use in paraplegic patients.

In restoring locomotion in those who are paraplegic, it is necessary to control the hip, knee and ankle joints and to maintain muscle activity to sustain the upright posture during standing and walking. Muscle fatigue is a major problem for restoration by FES. Ichie ${ }^{7}$ described several attempts to resolve the problem in this field: (1) controlling all the muscles which are related to locomotion and antigravity posture ${ }^{11}$ (2) introducing a withdrawal reflex for making locomotive motion $^{2}(3)$ decreasing loads to the antigravity muscles with an orthosis ${ }^{15}(4)$ introducing a closed-loop control system with sensors. ${ }^{1}$ We believe that reducing the amount of stimulation of the muscles can be achieved using a closed-loop control. Such a system requires joint position sensors that provide feedback information to the host computer to prevent falling, regulate the amount of stimulations and determine the timing of stimulations. Andrews ${ }^{1}$ has already developed a closedloop control system with surface electrodes. We have recently developed a new stimulator for a closed-loop control system with our percutaneous electrodes.

In addition, a hybrid FES with an anterior floor reaction type ankle-foot orthoses will provide several advantages in this field. (1) the brace alone will prevent knee collapse and thereby greatly reduce the need for electrical stimulation which induces muscle fatigue; (2) increasing the power can be supplied at the other joint by fixation of one joint of the two joint muscles; (3) decreasing the number of joints needing control; (4) easy to apply the closed-loop control system because the sensors such as electrogoniometers can be attached to the orthoses. ${ }^{7}$

Jaeger $^{8}$ has described that between 4.7 and $11.25 \%$ of all spinal cord injured persons in the USA might be potential users of a particular electrical stimulation technology. We expect that the demand of FES for paraplegic patients will increase in the near future, and we are working towards the development of practical FES systems which will help the independence our patients. 


\section{Acknowledgements}

The authors thank Professor BJ Andrews in University of Alberta for his co-operation in this study.

\section{References}

1 Andrews BJ et al. Hybrid FES orthosis incorporating closed and sensory feedback. J Biomed Eng 1988; 10: 189- 195.

2 Bajd T et al. Assessment of functional characteristics relevant to FES application in complete SCI patients. Proc 9th Intern Symp ECHE pp 129-140, 1987

3 Handa $Y$ et al. Studies on ultrafine intramuscular electrode and skin button for FES. Proc 10th Ann Intern Conf IEEE/EMBS pp 1686-1687, 1988.

4 Handa Y, Ohkubo K, Hoshimiya N. A portable multichannel FES system for restoration of motor function of the paralyzed extremities. Automedica 1989; 11: $221-231$.

5 Handa Y, Hoshimiya N, Iguchi Y, Oda T. Development of percutaneous intramuscular electrode for multichannel FES system. IEEE Trans Biomed Eng 1989; 36: 705-710.

6 Hoshimiya N, Handa Y. A master-slave type multichannel functional electrical stimulation (FES) system for the control of the paralyzed upper extremities. Automedica 1989; 11: 209220.
7 Ichie M. Restoration of locomotion by FES. Proc Rehabil Intern Seminar pp 1-12, 1990.

8 Jaeger RJ, Yarkony GM, Roth EJ, Lovell L. Estimating the user population of a simple electrical stimulation system for standing. Paraplegia 1990; 28: 505-511.

9 Kagaya $\mathrm{H}$. Therapeutic and functional electrical stimulation for paraplegics. J Jpn Ortop Assoc 1994; 68: 751-762.

10 Kantrowitz A. Electronic physiologic aids. A report of Maimonides Hospital pp 4-5, 1960.

11 Marsolais EB, Kobetic R. Functional electrical stimulation for walking in paraplegia. J Bone Joint Surg 1987; 69-A: $728-733$.

12 Marsolais EB, Kobetic R, Chizeck HJ, Jacobs JL. Orthoses and electrical stimulation for walking in complete paraplegia. $J$ Neuro Rehab 1991; 5: 13-22.

13 Scheiner A, Poland G, Marsolais B. Design and clinical application of a double helix electrode for functional electrical stimulation. IEEE Trans Biomed Eng 1994; 41: 425-431.

14 Shimada Y et al. Restoration of locomotion in paraplegics by functional electrical stimulation. Proc 1st Intern FES Symp pp 110-114, 1992.

15 Solomonow M et al. Gait performance of paraplegics ambulating with the reciprocating gait orthosis powered by electrical muscle stimulation. Proc 11th Intern Conf IEEE/EMBS p 1013, 1989.

16 Yarkony GM et al. Functional neuromuscular stimulation for standing after spinal cord injury. Arch Phys Med Rehabil 1990; 71: $201-206$. 